\title{
Constrained spectrum control using MPC
}

\author{
Ravi Gondhalekar, Colin N. Jones, Thomas Besselmann, Jean-Hubert Hours and Mehmet Mercangöz
}

\begin{abstract}
Well-known model predictive control (MPC) theory for constrained linear time-invariant (LTI) systems is extended to accommodate hard constraints and cost penalizations on the spectra of the system's output trajectories. Thus the proposed method facilitates enforcing constraints, and placing weights, on the harmonic content of input-, state- and output-trajectories, in addition to the usual constrained control objectives. The proposed methods are demonstrated by the example problem of reducing torsional vibrations in a drive-shaft.
\end{abstract}

Index Terms-Model predictive control; Constrained control; Spectrum control; Frequency cost-functionals

\section{INTRODUCTION}

Many aspects of constrained MPC theory are by now well-established [17,25,27,30], and MPC has proven effective for constrained control because it facilitates enforcing common geometric constraint objectives on states, inputs and outputs. However, constraints hamper designing the harmonic response of the closed-loop system, something readily achievable in an unconstrained setting using traditional loopshaping techniques. The aim of this paper is to establish a framework for constrained shaping of the output spectrum of a constrained system, using both cost penalizations, as well as by enforcing hard constraints, on the harmonic content of the output signal. To the authors' knowledge no method to enforce spectrum constraints yet exists. The contribution of this paper is a proposal for such a method.

There is a need to design and constrain the spectrum of constrained systems' responses. In [31], electromagnetic interference (EMI) emissions in switch-mode power supplies were suppressed by regulating the control signal's switching spectrum. Control of power converters with imposed load current spectra was considered in [11]. In [10], electrical power converters that produce sinusoidal output voltages with low harmonic distortion were considered. Similarities of $[10,11,31]$ are that the methods employ a filter, and using an MPC approach they penalize some function of the filter

Ravi Gondhalekar is with the Department of Mechanical Engineering and Frontier Research Base for Global Young Researchers, Graduate School of Engineering, Osaka University, Japan. Ravi gratefully acknowledges funding received through the Program of Promotion of Environmental Improvement to Enhance Young Researchers' Independence under the Special Coordination Funds for Promoting Science and Technology, Japan Ministry of Education, Culture, Sports, Science and Technology. ravi.gondhalekar@wakate.frc.eng.osaka-u.ac.jp

Colin N. Jones and Jean-Hubert Hours are with the Institute of Mechanical Engineering, École Polytechnique Fédérale de Lausanne, Switzerland. This research was partially funded from the European Union Seventh Framework Programme FP7/2007-2013 under grant agreement number FP7-ICT2009-4 248940. \{colin.jones, jean-hubert.hours\} depfl.ch

Thomas Besselmann and Mehmet Mercangöz are with ABB Switzerland Ltd., Corporate Research, Baden-Dättwil, Switzerland.

\{thomas.besselmann, mehmet.mercangoez\}@ech.abb.com output in order to affect a system's harmonic response. Filters are similarly employed here. In [20] an MPC approach and a variational constraint were utilized to limit switching signals to appliances on electrical power grids, for the purpose of reducing mechanical wear-and-tear. The approaches of $[10,11,20,31]$ are incapable of enforcing hard constraints on a system's output spectrum. However, it may be desirable to enforce such constraints, e.g. to rigorously comply with EMI emissions regulations [31]. Related matters, although less closely related, are the approach of [12] for constrained clipping of audio signals, the method of [36] for achieving frequency response specifications, the discussion in [28] on trade-offs of output current spectrum quality in predictive control of a permanent magnet synchronous machine, the need in [29] to perform frequency-dependent control actions for marine craft, and the need in [32] to regulate certain resonances when earthquake-proofing civil structures.

The proposed approach is described here via discretetime LTI systems, although it can be incorporated into a more general setting. A constrained LTI system is augmented by two LTI filters. One of the filters is employed to yield frequency cost-functionals which induce a coloration of the output spectrum. This is an extension of the continuoustime spectrum shaping results of $[19,24,33]$. The other filter is used to generate spectrum information that may then be constrained. The augmented system is LTI and may be made subject to well-known LTI-MPC methods.

Paper structure: The spectrum control problem is defined in Section II and the unconstrained spectrum control problem is solved in Section III. Spectrum constraints and the associated filter are discussed in Section IV, and in Section V an augmented state-space modeling framework is presented that allows the constrained spectrum control problem to be tackled using well-known LTI-MPC methods. In Section VI the proposed methods are demonstrated by numerical examples.

Notation: We denote by $\mathbb{R}$ the set of reals, by $\mathbb{C}$ the set of complex numbers, by $\mathbb{N}$ the set of non-negative integers, by $\mathbb{N}_{n}^{m}$ the set of consecutive non-negative integers $\{n, \ldots$, $m$ \}, by $I_{n}$ the $n \times n$ identity matrix, by 0 the zero matrix with appropriate dimension, by $\rho(A)$ the spectral radius of a matrix $A$, by $j$ the imaginary unit, and by $\left\{x_{k}\right\}_{k=n}^{m}$ a sequence of elements $x_{k} \forall k \in \mathbb{N}_{n}^{m}$.

\section{SPECTRUM CONTROL PROBLEM SETTING}

We consider the constrained discrete-time LTI system

$$
\begin{aligned}
& x_{k+1}=A x_{k}+B u_{k} \\
& E x_{k}+G u_{k} \leq W
\end{aligned}
$$

with step $k \in \mathbb{N}$, state $x \in \mathbb{R}^{n}$, and control input $u \in \mathbb{R}^{m}$. 
Assumption 1: The pair $(A, B)$ is stabilizable.

Assumption 2: At each step $k$ the state $x_{k}$ is known.

Assumption 3: $W>0$ (origin in interior of constraints)

We define a generalized output signal

$$
z_{k}:=C x_{k}+D u_{k} \in \mathbb{R}^{p} .
$$

Note that we consider control laws based on state-feedback, given Assumption 2. The output $z$ is not a measured system output, it is a mathematical construct defined solely to denote a signal of which to penalize and constrain the spectrum. As $z$ is a linear combination of $x$ and $u$ the proposed method provides a unified framework for constrained spectrum shaping of input-, state-, and measured output-trajectories, although measured outputs are ignored in this paper.

Assumption 4: $z_{k}=0 \quad \forall k<0$

In order to access the spectral components of $z$ we consider the discrete-time Fourier transform (DTFT) $\mathcal{Z}$ : $\mathbb{R} \rightarrow \mathbb{C}^{p}$ of output trajectory $\left\{z_{k}\right\}_{k=-\infty}^{+\infty}$, defined as follows:

$$
\mathcal{Z}(\omega):=\sum_{k=-\infty}^{+\infty} z_{k} \mathrm{e}^{-j \omega k} .
$$

One objective of this paper is to shape the DTFT spectrum $\mathcal{Z}(\omega)$ of the output trajectory using frequency weightings.

The second objective of this paper is to enforce hard constraints on the frequency components of the output trajectory. There are various desirable and implementable approaches, and which alternative is appropriate depends on the problem setting. In this paper we aim to constrain the magnitude of signal components of distinct frequencies $\omega_{i}, i \in \mathbb{N}_{1}^{r}$, e.g. the plant's resonant frequencies. Any number $r$ of distinct frequencies can be accommodated. We denote by $\tilde{\mathcal{Z}}\left(\omega_{i}, k\right)$, $\tilde{\mathcal{Z}}: \mathbb{R} \times \mathbb{N} \rightarrow \mathbb{C}^{p}$ a time-localized interpretation of $\mathcal{Z}\left(\omega_{i}\right)$, made at current time step $k$, based on past output values $z_{l}$, with $l$ in the temporal vicinity of $k$. The exact meaning of $\tilde{\mathcal{Z}}\left(\omega_{i}, k\right)$ is discussed in Section IV, but it is similar in spirit to, although more general than, the short-time Fourier transform (STFT) [6]. Then, employing this notion, the spectrum constraint objective is to enforce general mixed constraints of the form

$\Omega_{i}\left(x_{k}, u_{k},\left|\tilde{\mathcal{Z}}\left(\omega_{1}, k\right)\right|, \ldots,\left|\tilde{\mathcal{Z}}\left(\omega_{r}, k\right)\right|\right) \leq 0 \quad \forall i \in \mathbb{N}_{1}^{s}$

at all steps $k$, where the functions $\Omega_{i}$ specify the constraints, and $s$ is the total number of spectrum constraints. Having such mixed constraints allows state-dependent spectrum constraints, e.g., "at high speed do not tolerate large oscillations". Alternatively one could formulate spectrumdependent input constraints, e.g., "partially disregard pilot commands when the aircraft's phugoids are excited".

For simplicity, the proposed constrained spectrum control problem setting, and solution strategy, is presented in this paper via the problem of linear-quadratic regulation (LQR), although the described methods can straightforwardly tackle more general problems, like set-point/reference tracking, and also the robust, etc., variations of these. For initial state $x_{0}$ the objective then is frequency-weighted and spectrumconstrained discrete-time LQR Problem 5.
Problem 5: Determine:

$$
\begin{aligned}
\left\{u_{k}^{*}\left(x_{0}\right)\right\}_{k=0}^{\infty}: & :=\arg \min _{\left\{u_{k}\right\}_{k=0}^{\infty}} \frac{1}{2 \pi} \int_{-\pi}^{+\pi}\|\mathcal{F}(\omega) \mathcal{Z}(\omega)\|_{2}^{2} \mathrm{~d} \omega \\
& +\sum_{k=0}^{\infty}\left[x_{k}^{\top} Q x_{k}+u_{k}^{\top} R u_{k}+2 u_{k}^{\top} S x_{k}\right]
\end{aligned}
$$

subject to (2) and (3) for all $k \in \mathbb{N}$.

Weights $Q, R, S$ have their usual LQR interpretations and must satisfy $\left[\begin{array}{ll}Q & S^{\top} \\ S & R\end{array}\right] \succeq 0$. The weighting function $\mathcal{F}: \mathbb{R} \rightarrow$ $\mathbb{C}^{q \times p}$ is employed to induce a coloring of spectrum $\mathcal{Z}(\omega)$.

\section{UNCONSTRAINED SPECTRUM LQR}

In this section we derive the unconstrained solution to Problem 5. This result is very similar to the proposition described informally in [11], and is a discrete-time version of the result of $[19,24,33]$ for continuous-time unconstrained frequency-weighted LQR.

Suppose frequency weighting function $\mathcal{F}(\omega)$ is realized by

$$
\mathcal{F}(\omega):=\mathcal{C}_{1}\left(\mathrm{e}^{j \omega} I_{n_{\psi}}-\mathcal{A}_{1}\right)^{-1} \mathcal{B}_{1}+\mathcal{D}_{1},
$$

i.e. $\mathcal{F}(\omega)$ is the transfer function of discrete-time LTI filter

$$
\zeta_{k+1}=\mathcal{A}_{1} \zeta_{k}+\mathcal{B}_{1} z_{k} \quad, \quad \psi_{k}=\mathcal{C}_{1} \zeta_{k}+\mathcal{D}_{1} z_{k}
$$

with state $\zeta \in \mathbb{R}^{n_{\psi}}$. We make Assumption 6 about filter (4).

Assumption 6: $\psi_{k}=0 \quad \forall k<0, \quad \rho\left(\mathcal{A}_{1}\right)<1, \quad \zeta_{0}=0$

We next define the following parameters for later use:

$$
\begin{aligned}
\hat{x}_{k} & :=\left[\begin{array}{ll}
x_{k}^{\top} & \zeta_{k}^{\top}
\end{array}\right]^{\top}, \quad \hat{n}:=n+n_{\psi} \\
\hat{A} & :=\left[\begin{array}{cc}
A & 0 \\
\mathcal{B}_{1} C & \mathcal{A}_{1}
\end{array}\right], \quad \hat{B}:=\left[\begin{array}{c}
B \\
\mathcal{B}_{1} D
\end{array}\right] \\
\hat{Q} & :=\left[\begin{array}{cc}
Q+C^{\top} \mathcal{D}_{1}^{\top} \mathcal{D}_{1} C & C^{\top} \mathcal{D}_{1}^{\top} \mathcal{C}_{1} \\
\mathcal{C}_{1}^{\top} \mathcal{D}_{1} C & \mathcal{C}_{1}^{\top} \mathcal{C}_{1}
\end{array}\right] \\
\hat{R} & :=R+D^{\top} \mathcal{D}_{1}^{\top} \mathcal{D}_{1} D \\
\hat{S} & :=\left[\begin{array}{ll}
S+D^{\top} \mathcal{D}_{1}^{\top} \mathcal{D}_{1} C & D^{\top} \mathcal{D}_{1}^{\top} \mathcal{C}_{1}
\end{array}\right] .
\end{aligned}
$$

Assumption 7: $\hat{R} \succ 0, \quad \hat{Q} \succeq 0, \quad\left(\hat{Q}^{\frac{1}{2}}, \hat{A}\right)$ detectable.

Note that by Assumptions 1 and 6 the pair $(\hat{A}, \hat{B})$ is stabilizable. Then consider a matrix $\hat{P} \in \mathbb{R}^{\hat{n} \times \hat{n}}$ satisfying Riccati Eq. (5) and note that by Assumptions 1, 6 and 7 such a $\hat{P}$ exists and is unique. Further define feedback gain $\hat{K}$ according to (6). We can then state and prove Lemma 8 .

$$
\begin{aligned}
\hat{P}= & \hat{A}^{\top} \hat{P} \hat{A}+\hat{Q}- \\
& {\left[\hat{B}^{\top} \hat{P} \hat{A}+\hat{S}\right]^{\top}\left(\hat{R}+\hat{B}^{\top} \hat{P} \hat{B}\right)^{-1}\left[\hat{B}^{\top} \hat{P} \hat{A}+\hat{S}\right] } \\
\hat{K}:= & -\left(\hat{R}+\hat{B}^{\top} \hat{P} \hat{B}\right)^{-1}\left[\hat{B}^{\top} \hat{P} \hat{A}+\hat{S}\right]
\end{aligned}
$$

Lemma 8: The optimal solution to Problem 5, in the absence of constraints (2) and (3), is given by

$$
u_{k}=\hat{K} \hat{x}_{k} \quad \forall k \in \mathbb{N} .
$$

Proof: Let $\Psi(\omega), \Psi: \mathbb{R} \rightarrow \mathbb{C}^{q}$ denote the DTFT of the output $\psi$ of filter (4). Then by Parseval's Theorem [3,7,21]:

$$
\begin{aligned}
\sum_{k=0}^{+\infty} \psi_{k}^{\top} \psi_{k} & =\sum_{k=-\infty}^{+\infty}\left\|\psi_{k}\right\|_{2}^{2}=\frac{1}{2 \pi} \int_{-\pi}^{+\pi}\|\Psi(\omega)\|_{2}^{2} \mathrm{~d} \omega \\
& =\frac{1}{2 \pi} \int_{-\pi}^{+\pi}\|\mathcal{F}(\omega) \mathcal{Z}(\omega)\|_{2}^{2} \mathrm{~d} \omega .
\end{aligned}
$$


Thus the unconstrained solution to Problem 5 is equal to

$\left\{u_{k}^{*}\left(x_{0}\right)\right\}_{k=0}^{\infty}$

$=\arg \min _{\left\{u_{k}\right\}_{k=0}^{\infty}} \sum_{k=0}^{\infty}\left[x_{k}^{\top} Q x_{k}+u_{k}^{\top} R u_{k}+2 u_{k}^{\top} S x_{k}+\psi_{k}^{\top} \psi_{k}\right]$

$=\arg \min _{\left\{u_{k}\right\}_{k=0}^{\infty}} \sum_{k=0}^{\infty}\left[\hat{x}_{k}^{\top} \hat{Q} \hat{x}_{k}+u_{k}^{\top} \hat{R} u_{k}+2 u_{k}^{\top} \hat{S} \hat{x}_{k}\right]$

with $\hat{x}_{k+1}=\hat{A} \hat{x}_{k}+\hat{B} u_{k}$, for which (7) provides the solution by well-known LQR theory.

\section{FORMULATING SPECTRUM CONSTRAINTS}

In this section we address the formulation of spectrum constraints (3). For each frequency $\omega_{i}$ that we wish to constrain we employ a frequency sampling filter [4], that at each step $k$ receives the output $z_{k}$ as an input, and that has a transfer function with a magnitude response of unity at $\omega_{i}$, and zero otherwise. The filter output is then constrained. Ideal filters are not realizable, thus in practice it is not possible to constrain singular frequencies, but generally spectrum constraints are enforced across the filter's passband $^{1}$. Note, however, that in practice it may be preferable to use non-ideal filters, with a substantial pass-band, because that allows constraining signals in a band of frequencies using just one filter. As mentioned above, various other constraint strategies are possible, and the preferred choice depends on the application details. Frequency "binning" or integral spectrum constraints are an alternative to the strategy presented here (see Section VII).

In this paper we employ LTI filters of the form

$$
\xi_{k+1}=\mathcal{A}_{2} \xi_{k}+\mathcal{B}_{2} z_{k} \quad, \quad \phi_{k}=\mathcal{C}_{2} \xi_{k}+\mathcal{D}_{2} z_{k}
$$

with state $\xi \in \mathbb{R}^{n_{\phi}}$ and output $\phi \in \mathbb{R}^{q_{\phi}}$, and we make the following assumption.

Assumption 9: $\phi_{k}=0 \quad \forall k<0, \quad \rho\left(\mathcal{A}_{2}\right)<1, \quad \xi_{0}=0$

We wish to formulate spectrum constraint (3) in a general form that is tractable for optimization in MPC, so henceforth we restrict the discussion to the formulation of (convex) second-order cone (SOC) constraints [9]. The goal is then to design control laws such that, in addition to geometric constraints (2), system (1) satisfies SOC spectrum constraints

$$
\left\|\Theta_{i}\left[\begin{array}{lll}
x_{k}^{\top} & u_{k}^{\top} & \phi_{k}^{\top}
\end{array}\right]^{\top}+\theta_{i}\right\|_{2} \leq \gamma_{i}^{\top}\left[\begin{array}{lll}
x_{k}^{\top} & u_{k}^{\top} & \phi_{k}^{\top}
\end{array}\right]^{\top}+\delta_{i}
$$

for all $i \in \mathbb{N}_{1}^{s}$, where $s$ is the number of constraints, and entities $\Theta_{i}, \theta_{i}, \gamma_{i}, \delta_{i}$ are design parameters. Placing SOC constraints on the spectrum allows us to formulate a wide variety of convex constraints (3), and an example is given at the end of this section. Note that SOC constraint (9) subsumes linear and quadratic constraints [9]. To be meaningful for constrained LQR we make Assumption 10.

Assumption 10: $\left\|\theta_{i}\right\|_{2} \leq \delta_{i} \forall i \in \mathbb{N}_{1}^{s}$ (origin in interior)

There are a large number of design decisions to be made when choosing filter (8) and constraints (9), and a thorough

\footnotetext{
${ }^{1}$ The term "pass-band" is a little misleading in this context, because signals that fall within the pass-band are those constrained.
}

discussion is beyond the scope of this paper. Outlined below are some basic design considerations.

Finite impulse response (FIR) filters are attractive on the one hand. They are simple to implement, and inherently stable, because $\mathcal{A}_{2}$ is nilpotent. The length of time the output $z_{k}$ can affect the spectrum constraint is determined by the FIR filter order, because the impulse response of an $M$ th order FIR filter is exactly $M+1$ steps long. Furthermore, any number of $M$ th order FIR frequency sampling filters can be implemented with a common state $\xi$, where the state $\xi$ is simply a shifting memory bank of past output values, and each filter occupies one row of output matrices $\mathcal{C}_{2}$ and $\mathcal{D}_{2}$. For example filters based on the discrete Fourier transform (DFT) [21,35] could be used. This is the approach employed in Section VI-B, and described below. Note that the DFT is a complex mapping, but linear, so the real and imaginary parts are easily determined separately. Simple magnitude constraints imposed on the outputs of DFT based filters are then implemented using quadratic constraints. Magnitude constraints that depend linearly on the state or input are implemented using SOC constraints. Approximations of magnitude constraints can be implemented using only linear constraints. Alternatively, FIR filters based on other transforms, e.g. the discrete cosine transform (DCT) and discrete sine transform (DST) [1,26,34], could be employed, as is done in Section VI-A. The DCT and DST determine the frequency components in terms of cosine and sine basis functions, respectively, and are real mappings. While derivatives of the DFT, the DCT and DST have higher spectral resolution for the same length of input data sequence.

FIR filters with higher selectivity require higher filter orders (see Fig. 1), which leads to larger state dimension $n_{\phi}$. In contrast infinite impulse response (IIR) filters can be designed to be more selective for smaller filter orders and state dimensions. However, for a fixed filter order, increasing the selectivity means that $\rho\left(\mathcal{A}_{2}\right) \rightarrow 1$. This implies that the length of time that historical outputs $z_{l}$ can significantly affect the current filter output $\phi_{k}$ becomes longer. This limitation is analogous to that of FIR filters - to distinguish two signals of increasingly similar frequency requires observing the two signals for increasing lengths of time.

Windows are a key element to consider when determining spectra from finite-length signal trajectories, and these attempt to eliminate spectral "pollution" introduced by the finite signal length. The authors have no rigorous design advice, but the Hamming window, proven effective in signal processing, has demonstrated to be far superior to a simple rectangular window, and therefore is employed in Section VI.

In Fig. 1 four FIR filters' responses, of increasing filter order, are plotted. The filters are based on the DCT, and for simplicity are set to sample a signal of a quarter the sampling frequency. The reduction of side-lobes due to the Hamming window is clear, but this comes at the expense of lower selectivity, for a filter of the same order.

In Section VI we make use only of FIR filters and Hamming windows, although the proposed methods hold for both FIR and IIR filters, and for any window scheme. 

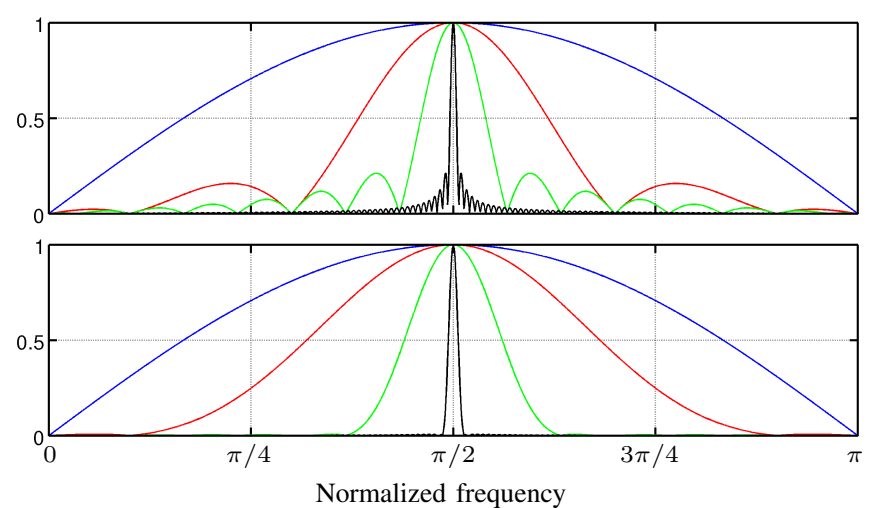

Fig. 1. Magnitude response of $M$ th order FIR frequency sampling filter based on the DCT with $M \in\{3,11,31,301\}$. Larger $M \Longrightarrow$ more selective filter. Top: Rectangular window. Bottom: Hamming window.

As a demonstration of the proposed approach, suppose $z$ is scalar, and that we need a simple magnitude constraint, based on the DFT, on output signals of frequency $\bar{\omega}$, and we believe that at each step $k$ considering a 4 -step historical output sequence $\left\{z_{l}\right\}_{l=k-3}^{k}$ gives a good trade-off between filter selectivity and impulse response length (delay). Thus

$$
\begin{aligned}
\tilde{\mathcal{Z}}(\bar{\omega}, k) & :=\sum_{l=0}^{3} z_{k+l-3} \mathrm{e}^{-j l \bar{\omega}} \\
& =\sum_{l=0}^{3}(\cos (l \bar{\omega})-j \sin (l \bar{\omega})) z_{k+l-3} \\
& =: \alpha_{1}-j \alpha_{2}
\end{aligned}
$$

and for some $\beta$ (positive scalar real) we want to enforce

$$
|\tilde{\mathcal{Z}}(\bar{\omega}, k)|^{2}=\alpha_{1}^{2}+\alpha_{2}^{2} \leq \beta .
$$

We implement this by defining

$$
\begin{aligned}
& \mathcal{A}_{2}:=\left[\begin{array}{lll}
0 & 0 & 0 \\
1 & 0 & 0 \\
0 & 1 & 0
\end{array}\right] \quad \mathcal{B}_{2}:=\left[\begin{array}{l}
1 \\
0 \\
0
\end{array}\right] \\
& \mathcal{C}_{2}:=\left[\begin{array}{rrr}
\cos (2 \bar{\omega}) & \cos (1 \bar{\omega}) & \cos (0 \bar{\omega}) \\
-\sin (2 \bar{\omega}) & -\sin (1 \bar{\omega}) & -\sin (0 \bar{\omega})
\end{array}\right] \\
& \mathcal{D}_{2}:=\left[\begin{array}{r}
\cos (3 \bar{\omega}) \\
-\sin (3 \bar{\omega})
\end{array}\right]
\end{aligned}
$$

and subsequently enforce the quadratic constraint

$$
\phi_{k}^{\top} \phi_{k} \leq \beta \text {. }
$$

The above derivation uses a rectangular window, but any non-rectangular window can easily be incorporated into (10).

In the example above (10) is the STFT of $z$, and thus constraint (11) is a constraint on the spectrogram [6] of $z$.

Quadratic constraint (11) can be approximated arbitrarily closely by polyhedral constraints on $\phi_{k}$. Such polyhedral constraints facilitate formulating common linear programming (LP) and quadratic programming (QP) problems [9].

\section{FULl LTI STATE-SPACE MODEL FOR MPC}

In this section we construct a fully augmented statespace model that permits the formulation of Problem 5 in a standard way. We define

$$
\begin{aligned}
\bar{x}_{k} & :=\left[\begin{array}{ccc}
x_{k}^{\top} & \zeta_{k}^{\top} & \xi_{k}^{\top}
\end{array}\right]^{\top} \\
\bar{A} & :=\left[\begin{array}{ccc}
A & 0 & 0 \\
\mathcal{B}_{1} C & \mathcal{A}_{1} & 0 \\
\mathcal{B}_{2} C & 0 & \mathcal{A}_{2}
\end{array}\right], \quad \bar{B}:=\left[\begin{array}{c}
B \\
\mathcal{B}_{1} D \\
\mathcal{B}_{2} D
\end{array}\right] \\
\bar{Q} & :=\operatorname{diag}(\hat{Q}, 0), \quad, \quad \bar{R}:=\hat{R}, \quad \bar{S}:=\left[\begin{array}{ll}
\hat{S} & 0
\end{array}\right]
\end{aligned}
$$

and consider augmented LTI system (12):

$$
\bar{x}_{k+1}=\bar{A} \bar{x}_{k}+\bar{B} u_{k}
$$

Constraints (2) and (9) are straightforwardly formulated as SOC constraints on $\left[\bar{x}_{k}, u_{k}\right]^{\top}$. The solution to Problem 5 is then equal to the solution to Problem 11.

Problem 11: Determine:

$$
\begin{aligned}
\left\{u_{k}^{*}\left(\bar{x}_{0}\right)\right\}_{k=0}^{\infty}:= & \arg \min _{\left\{u_{k}\right\}_{k=0}^{\infty}} \\
& \sum_{k=0}^{\infty}\left[\bar{x}_{k}^{\top} \bar{Q} \bar{x}_{k}+u_{k}^{\top} \bar{R} u_{k}+2 u_{k}^{\top} \bar{S} \bar{x}_{k}\right]
\end{aligned}
$$

subject to (2) and (9) for all $k \in \mathbb{N}$.

Lemma 12: The pair $(\bar{A}, \bar{B})$ is stabilizable if and only if the pair $(A, B)$ is stabilizable.

Lemma 13: $\bar{R} \succ 0, \bar{Q} \succeq 0,\left(\bar{Q}^{\frac{1}{2}}, \bar{A}\right)$ detectable.

Lemmata 12 and 13 are direct consequences of Assumptions $1,6,7$, and 9 , and no further proofs are provided.

Problem 11 can now be tackled using well known LTI MPC theory [17,25,27]. MPC details are not discussed in this paper, because given the modeling framework presented in this section, the MPC methods employed in the examples in Section VI are standard. As mentioned above, Problems 5 and 11 are LQR problems, however, the proposed frequency cost-functionals and spectrum constraint mechanism can straightforwardly be incorporated into more general problems, e.g. tracking problems [14,22] (as we do in Section VI), and also robust versions of these [2,15,18,23] (robustness is ignored throughout this paper).

In MPC design we frequently make use of invariant sets $[5,13,16]$, because these are generally required to give guarantees of recursive feasibility, stability, optimality and robustness [27]. Invariant sets for systems subject to SOC constraints cannot be determined (at present), thus it may be desirable to limit oneself to either linear or quadratic constraints (2) and (9), for which invariant sets may be characterized. The framework proposed here generally leads to models with high state dimension $\bar{n}$, even if the original system's state dimension $n$ is small. Thus the complexity of computing polyhedral invariant sets may be prohibitive. However, in some situations it is not required to compute invariant sets in $\mathbb{R}^{\bar{n}}$, instead invariant sets of lower dimension may be employed (detailed discussion omitted for brevity).

\section{NUMERICAL EXAMPLES}

\section{A. An illustrative toy example}

We consider the system $x_{k+1}=u_{k}, z_{k}=x_{k}$ with scalar $x$ and $u$, and no constraints (2). The reason for choosing the trivial dynamics is to demonstrate the effects and idiosyncrasies of spectrum weighting and spectrum constraining, 

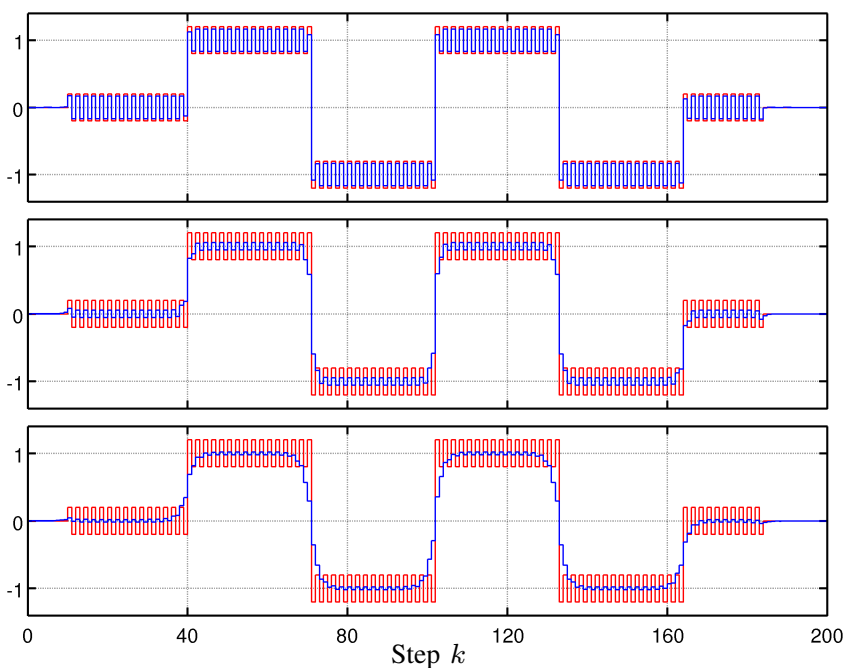

Fig. 2. Toy example: Low (top), medium (center) and high (bottom) weighting on high-frequency component. State $x$ : blue. Reference: red.

and differences between the two, without mixing in spectral coloration due to the plant's intrinsic transfer characteristics. The control objective is to track a state-input reference trajectory that is known a priori. This system can clearly be controlled to track any (known) reference trajectory, without errors, using any length of prediction horizon. The reference trajectory is 200 steps long, with two periods of a lowfrequency signal of large amplitude, superimposed onto a high-frequency signal of 2-step period length and small amplitude, and with a constant beginning and end. The statereference trajectory is plotted in red in Figs. 2 to 6 . We choose a prediction horizon length of 30 , and weighting matrices $R=Q=1, S=0$.

Filters (4) and (8) are 3rd order FIR filters based on the DCT in combination with a Hamming window (details omitted for brevity). Basically, at step $k$ the filters send the 4-step output trajectory $\left\{z_{l}\right\}_{l=k-3}^{k}$ through a Hamming
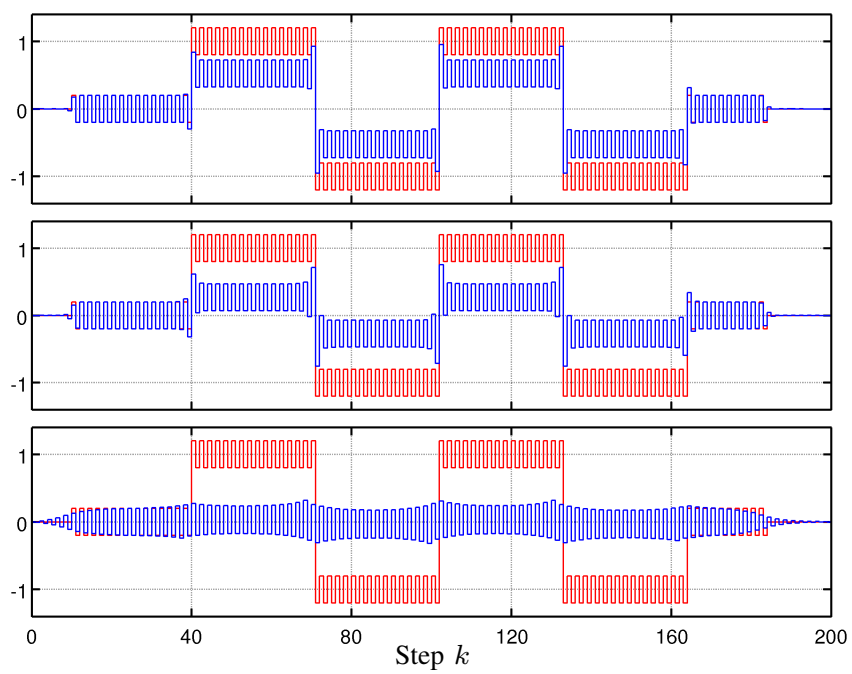

Fig. 3. Toy example: Low (top), medium (center) and high (bottom) weighting on DC component. State $x$ : blue. Reference: red.
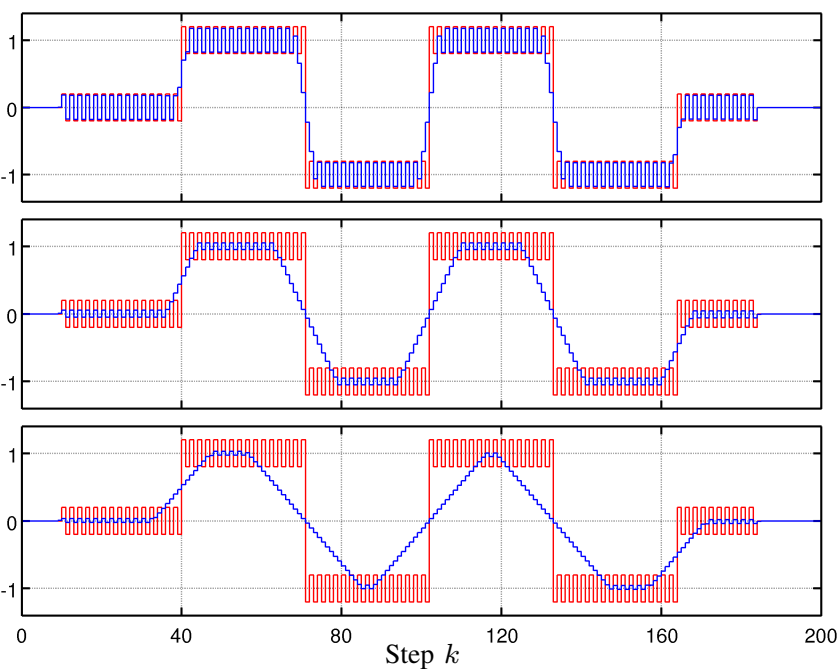

Fig. 4. Toy example: Loose (top), medium (center) and tight (bottom) constraint on high-frequency component. State $x$ : blue. Reference: red.

window, compute the DCT, then either the highest frequency component, or the DC value, is penalized and constrained, depending on the case, as explained below.

Plotted in Figs. 2 and 4 are the state trajectories resulting from spectrum weighting (no constraints) and spectrum constraints (no weighting), respectively, on the high-frequency component. Three weightings and constraints each are presented, and these were tuned to give a similar residual highfrequency component (tuning details omitted). The highfrequency component is clearly smoothed out in Fig. 2, while large transitions are performed when needed. In contrast, in Fig. 4 the constraints effectively limit the slew-rate, and large reference transitions lead to large tracking errors.

Plotted in Figs. 3 and 5 are the state trajectories resulting from spectrum weighting (no constraints) and spectrum constraints (no weighting), respectively, on the DC component. Three weightings and constraints each are presented, and
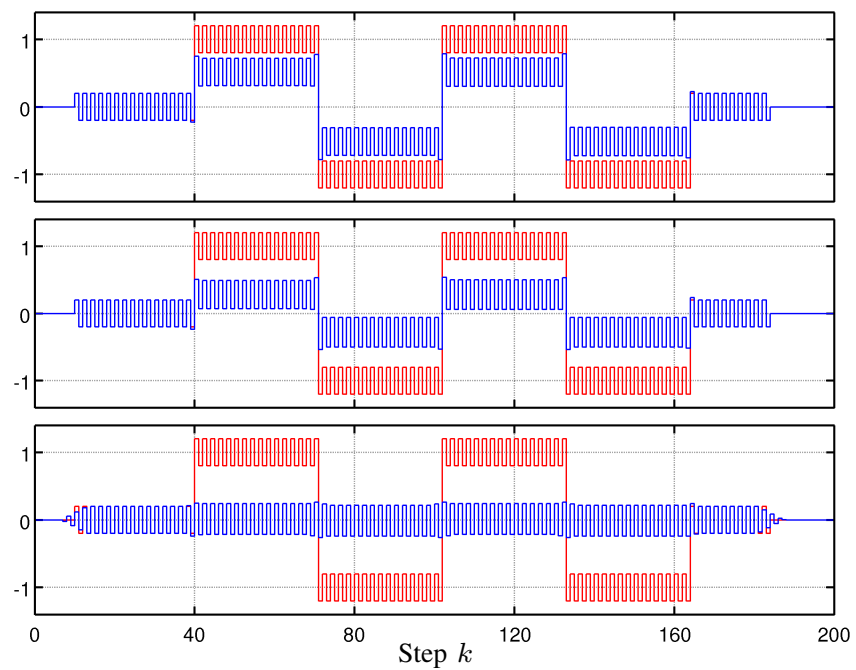

Fig. 5. Toy example: Loose (top), medium (center) and tight (bottom) constraint on DC component. State $x$ : blue. Reference: red. 

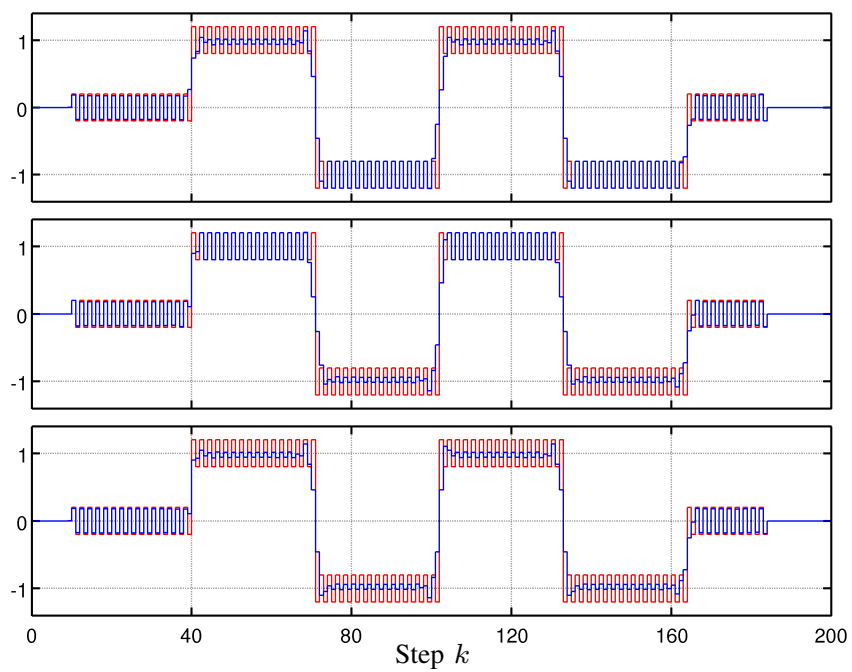

Fig. 6. Toy example: State-dependent spectrum constraints on highfrequency component. State $x$ : blue. Reference: red.

these were tuned to give similar residual DC components (tuning details omitted). The DC component is clearly removed in both cases. Interestingly, the weighting introduces significantly more high-frequency "ringing" before the highfrequency reference trajectory starts, and after it has ended. Furthermore, Fig. 3 displays Gibbs phenomenon. Note, however, that it is quite coincidental that the other figures do not display Gibbs phenomenon. In the authors' experience Gibbs phenomenon frequently occurs without careful filter design, and especially so for certain combinations of prediction horizon length, filter order and window function. Note that in this example we are penalizing and constraining a single frequency component - in general a more even weighting and/or constraining avoids the "water-bed effect" of shifting signal energy to other frequencies.

Plotted in Fig. 6 are three examples with state-dependent spectrum constraints - the high-frequency constraint is tighter as the state is larger (top), as the state is smaller (middle), and as $|x|$ is larger (bottom). Using the proposed framework it is not possible to specify a state-dependent spectrum constraint that tightens when $|x|$ becomes smaller, as such a constraint is non-convex. Note that the convex state-dependent spectrum constraints of Fig. 6 are easily implemented in the LTI setting with mixed constraints (9). In contrast, state-dependent weightings cannot be accommodated in an LTI setting. They could be approximated in an LTI setting using mixed linear costs, but to implement rigorously may require a PWA hybrid-systems approach.

\section{B. Torsional vibration control example}

The objective in this example is torsional vibration control in a drive-shaft. Torsional vibrations are oscillations of the relative angular positions and velocities of the elements of a shaft. They often occur unexpectedly and can cause extensive damage $[8,37]$. Note that the purpose of this example is to demonstrate the spectrum weighting and spectrum constraint

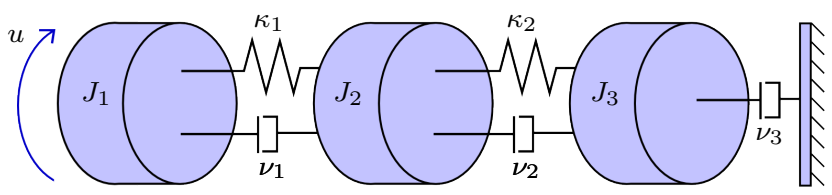

Fig. 7. Vibration control example: Simple drive-shaft model.

method, and that the presented results are not the optimal tuning for suppression of torsional oscillations in a drive-shaft.

A simple model of a drive-shaft is depicted in Fig. 7, and consists of three concentric rotating discs connected by torsional spring-damper units. The control input is a (scalar) torque applied to disc 1 , and disc 3 is connected to a fixed load by a torsional damper. The following parameters are used: $J_{1}=J_{2}=10 \mathrm{~kg} \mathrm{~m}^{2}, J_{3}=10 \cdot J_{1}, \kappa_{1}=10^{4} \mathrm{Nm} / \mathrm{rad}$, $\kappa_{2}=10 \cdot \kappa_{1}, \nu_{1}=\nu_{2}=\nu_{3}=0.1 \mathrm{Nm} \mathrm{s} / \mathrm{rad}$. We denote by $\theta_{1}, \theta_{2}, \theta_{3}$ the angular positions of the discs. The absolute angular positions are irrelevant here, of importance are the relative angles between adjacent discs. The plant can be modeled by a continuous-time LTI system with state and output

$$
\begin{aligned}
& x:=\left[\begin{array}{lllll}
\left(\theta_{1}-\theta_{2}\right) & \left(\theta_{2}-\theta_{3}\right) & \dot{\theta}_{1} & \dot{\theta}_{2} & \dot{\theta}_{3}
\end{array}\right]^{\top} \in \mathbb{R}^{5} \\
& z:=10^{4}\left(\theta_{1}-\theta_{2}\right) \in \mathbb{R} .
\end{aligned}
$$

The units of output $z$ are $\left[10^{-4} \mathrm{rad}\right]$ (tenths of milli-rad). The continuous-time system is sampled at $250 \mathrm{~Hz}$ using a zero-order hold on the input. The subsequent application of MPC is performed on the resulting discrete-time system.

The control objective is to perform a unit step change in the output, while avoiding exciting the normal modes of vibration of the system. The open-loop system has two resonances, at roughly $5 \mathrm{~Hz}$ and $17 \mathrm{~Hz}-$ see Fig. 8 .

We first employ a standard MPC strategy with cost matrices $Q=\operatorname{diag}(300,0,0,0,0), R=1, S=0$, and a prediction horizon length of 30 . The output response is plotted in blue in Figs. 9 and 10. Clearly the response is highly oscillatory, with excitation predominantly of the $17 \mathrm{~Hz}$ resonance.

We next introduce a frequency weighting (no spectrum constraints), to specifically target the $17 \mathrm{~Hz}$ resonance. We choose filter (4) to be a 3rd order Butterworth filter, tuned to $17 \mathrm{~Hz}$, with the following state-space matrices:

$\begin{aligned} \mathcal{A}_{1} & =\left[\begin{array}{cccccc}5.25 & -2.97 & 1.85 & -0.67 & 0.26 & -0.18 \\ 4 & 0 & 0 & 0 & 0 & 0 \\ 0 & 2 & 0 & 0 & 0 & 0 \\ 0 & 0 & 2 & 0 & 0 & 0 \\ 0 & 0 & 0 & 1 & 0 & 0 \\ 0 & 0 & 0 & 0 & 0.25 & 0\end{array}\right] \\ \mathcal{B}_{1} & =\left[\begin{array}{lllllll}0.0625 & 0 & 0 & 0 & 0 & 0\end{array}\right]^{\top} \\ \mathcal{C}_{1} & =\left[\begin{array}{llllll}369 & -261 & 130 & -34 & 19 & -30\end{array}\right] \cdot 10^{-4} \\ \mathcal{D}_{1} & =4.39 \cdot 10^{-4} .\end{aligned}$

The filter's magnitude response is plotted in red in Fig. 8. Note that the frequency weighting can be tuned by scaling the filter's output matrices $\mathcal{C}_{1}$ and $\mathcal{D}_{1}$. Plotted in Fig. 9 are the input and output trajectories for two different Butterworth 


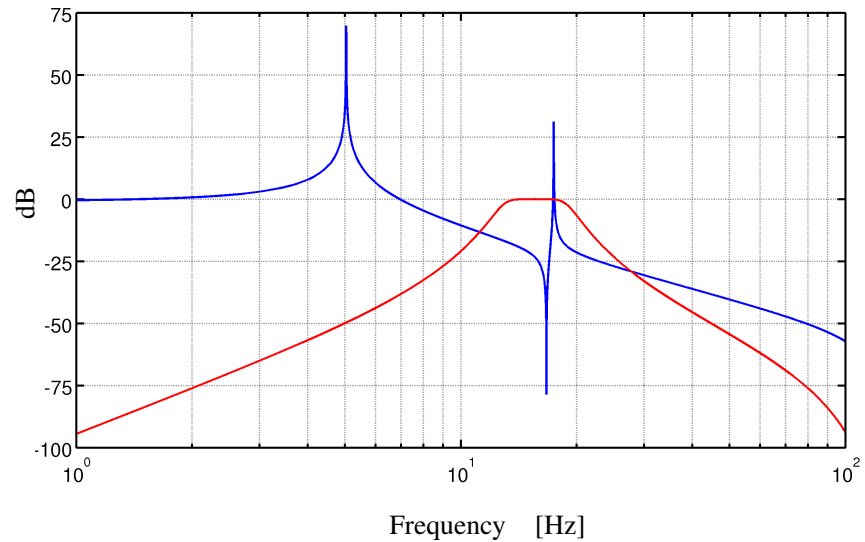

Fig. 8. Vibration control example: Magnitude frequency response of plant (blue) and 3rd order Butterworth frequency weighting filter $\mathcal{F}(\omega)$ (red).

filter weightings, one small (red), one large (black). It can be seen that an increased Butterworth filter weighting more effectively suppresses the $17 \mathrm{~Hz}$ oscillations. However, this comes at the expense of higher overshoots.

We next introduce spectrum constraints (no frequency weighting), again targeting the $17 \mathrm{~Hz}$ resonance. We choose filter (8) to be based on the DFT using a history of 41 output values, and enforce quadratic spectrum constraints analogous to the example of (11). The resulting input and output trajectories are plotted in red in Fig. 10. The oscillations in the output are clearly removed very effectively, but are not removed completely as in the case when employing a heavy frequency weighting. An advantage of spectrum constraints
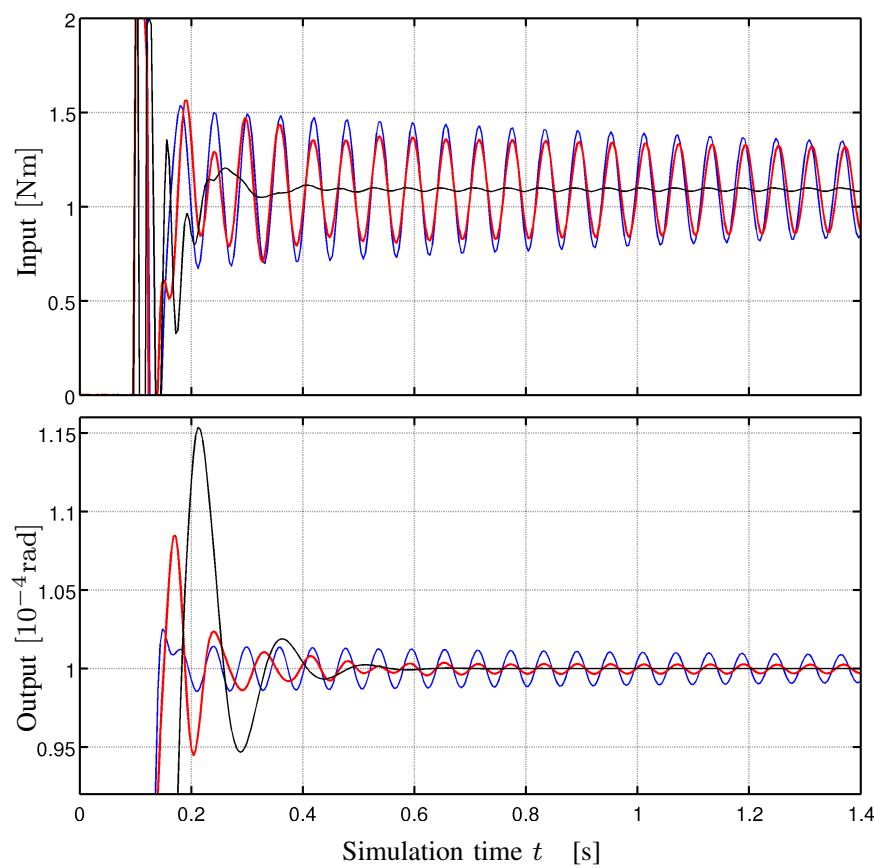

Fig. 9. Vibration control example: Input and output trajectories for three different MPC schemes: Standard MPC (blue), Butterworth-MPC with low weight (red), and Butterworth-MPC with high weight (black). The reference signal is a unit step change in output at $t=0.1 \mathrm{~s}$. over frequency weighting is the absence of an overshoot, but the price for this is a somewhat sluggish step response.

This example demonstrates that both frequency costfunctionals as well as the proposed hard spectrum constraints are very useful elements in the repertoire of tools available to manipulate the response of a constrained system.

\section{CONCLUSION AND FUTURE WORK}

In this paper the novel concept of constrained spectrum control was considered, and an MPC approach for the constrained shaping of a constrained LTI system's output spectra was presented. The salient ingredients are two filters, one that specifies frequency cost-functionals, and another that provides a time-localized interpretation of the output spectra which to constrain. The constrained spectrum control problem could then be re-cast into a usual LTI framework and well-known MPC theory applied.

This paper is the first report on a new research direction, and there is a large number of opportunities to further explore the described problem setting and proposed solution approach. The extension to spectrum control of more general classes of systems, e.g. nonlinear, time-varying, hybrid, etc., is obvious. However, there are two fundamental areas that hold important and unanswered questions, irrespective of the plant details - recall that the two filters are LTI.

The first is the question of what classes of spectrum constraints are desirable and implementable. The method as presented can be employed to constrain the magnitude of signals of a finite selection of frequencies using frequency sampling filters. However, linear/quadratic integral magnitude constraints, and also phase constraints, are tractable
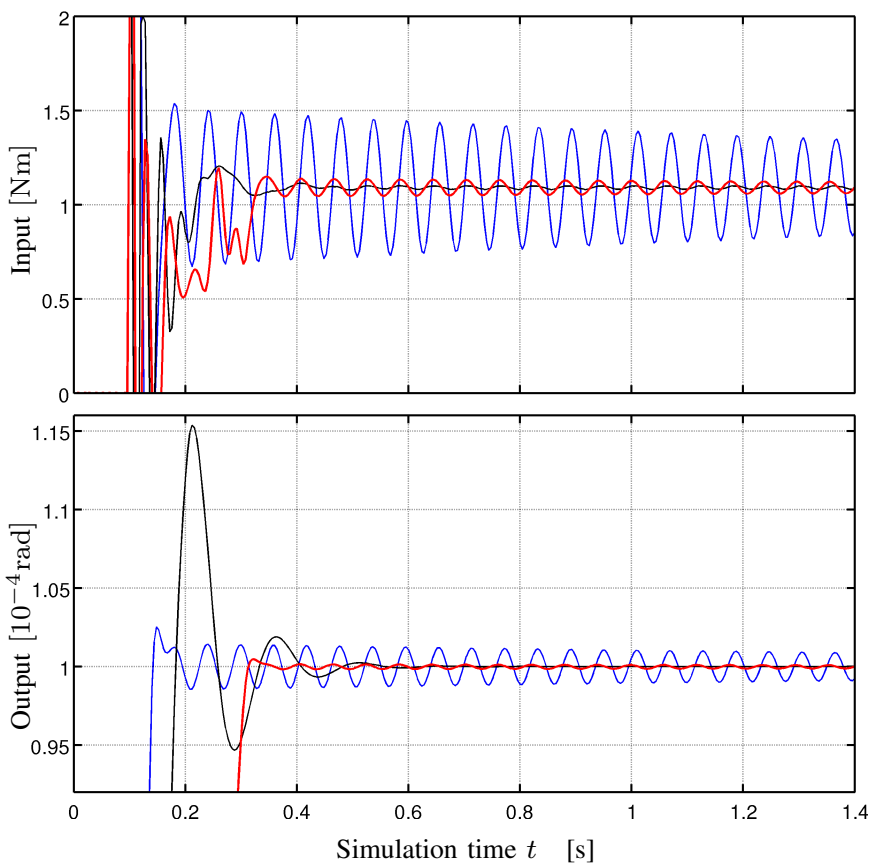

Fig. 10. Vibration control example: Input and output trajectories for three different MPC schemes: Standard MPC (blue, same as Fig. 9), ButterworthMPC with high weight (black, same as Fig. 9), and spectrum-constrained MPC (red). The reference signal is a unit step change in output at $t=0.1 \mathrm{~s}$. 
alternatives. A more complete picture of the possibilities, with accompanying applications, is required.

The second is the issue of optimal filter and window design for the different possibilities of spectrum specification. The use of DFT/DCT-based filters in Section VI was for simplicity, and because they are well understood. The Hamming window was chosen because it is regarded as effective by the signal processing community. A plethora of possible filter/window substitutes exists and a rigorous design methodology must be developed - tailored to the purpose of control.

\section{ACKNOWLEDGMENT}

The authors thank Alexander Domahidi (ETHZ), Shahab Kaynama (UBC), Dr. Haitham Hindi (PARC) and Prof. Robert Bitmead (UCSD) for enlightening discussions and for suggesting references.

\section{REFERENCES}

[1] N. Ahmed, T. Natarajan \& K. R. Rao. Discrete Cosine Transform. IEEE Trans. Computers, C-23(1):90-93, 1974.

[2] A. Bemporad \& M. Morari. Robust model predictive control: A survey. Robustness in Identification \& Control, 245:207-226, 1999.

[3] D. S. Bernstein. Matrix Mathematics: Theory, Facts, and Formulas with Application to Linear Systems Theory. Princeton, 2005.

[4] R. R. Bitmead \& B. D. O. Anderson. Adaptive frequency sampling filters. IEEE Trans. Circuits \& Systems, CAS-28(6):524-534, 1981.

[5] F. Blanchini. Set invariance in control. Automatica, 35(11):1747-1767, 1999.

[6] B. Boashash. Time Frequency Signal Analysis and Processing. Elsevier, 2003.

[7] M. L. Boas. Mathematical Methods in the Physical Sciences. Wiley, 1966.

[8] A. P. Bovsunovskii, O. Y. Chernousenko, E. V. Shtefan \& D. A. Bashta. Fatigue damage and failure of steam turbine rotors by torsional vibrations. Strength of Materials, 42:108-113, 2010.

[9] S. P. Boyd \& L. Vandenberghe. Convex Optimization. Cambridge, 2004.

[10] P. Cortés, G. Ortiz, J. I. Yuz, J. Rodríguez, S. Vazquez \& L. G. Franquelo. Model predictive control of an inverter with output $l c$ filter for UPS applications. IEEE Trans. Industrial Electronics, 56(6):1875$1883,2009$.

[11] P. Cortés, J. Rodríguez, D. E. Quevedo \& C. Silva. Predictive current control strategy with imposed load current spectrum. IEEE Trans. Power Electronics, 23(2):612-618, 2008.

[12] B. Defraene, T. van Waterschoot, M. Diehl \& M. Moonen. A fast projected gradient optimization method for real-time perception-based clipping of audio signals. In Proc. IEEE Int. Conf. Accoustics, Speech \& Signal Processing, pp. 333-336, Prague, Czech Republic, 2011.

[13] C. E. T. Dórea \& J.-C. Hennet. $(A, B)$-Invariant polyhedral sets of linear discrete-time systems. Journal of Optimization Theory and Applications, 103(3):521-542, 1999.

[14] A. Ferramosca, D. Limon, I. Alvarado, T. Alamo \& E. F. Camacho. MPC for tracking with optimal closed-loop performance. Automatica, 45(8):1975-1978, 2009.
[15] H. Fukushima \& R. R. Bitmead. Robust constrained model predictive control using closed-loop prediction. In Proc. AACC American Control Conf., pp. 2511-2516, Denver, CO, USA, 2003.

[16] E. G. Gilbert \& K. T. Tan. Linear systems with state and control constraints: The theory and application of maximal output admissible sets. IEEE Trans. Automatic Control, 36(9):1008-1020, 1991.

[17] G. C. Goodwin, M. M. Seron \& J. A. De Doná. Constrained Control and Estimation: An Optimisation Approach. Springer, 2005.

[18] P. J. Goulart, E. C. Kerrigan \& J. M. Maciejowski. Optimization over state feedback policies for robust control with constraints. Automatica, 42(4):523-533, 2006

[19] N. K. Gupta. Frequency-shaped cost functionals - extension of linearquadratic-gaussian design methods. Journal of Guidance, Control, and Dynamics, 3(6):529-535, 1980.

[20] H. Hindi, D. Greene \& C. Laventall. Coordinating regulation and demand response in electric power grids using multirate model predictive control. In Proc. IEEE PES Conf. Innovative Smart Grid Technologies, Gaithersburg, MA, USA, 2011.

[21] G. James. Advanced Modern Engineering Mathematics. Addison Wesley, 1993.

[22] D. Limon, I. Alvarado, T. Alamo \& E. F. Camacho. MPC for tracking piecewise constant references for constrained linear systems. Automatica, 44(9):2382-2387, 2008.

[23] J. Löfberg. Approximations of closed-loop minimax MPC. In Proc. IEEE Conf. Decision \& Control, pp. 1438-1442, Maui, HI, USA, 2003.

[24] L. Lublin \& M. Athans. Linear quadratic regulator control. In W. S. Levine, editor, The Control Handbook: Control System Advanced Methods, chapter 17. CRC Press, 2 edition, 2011.

[25] J. M. Maciejowski. Predictive Control with Constraints. Prentice Hall, 2002.

[26] S. A. Martucci. Symmetric convolution and the discrete sine and cosine transforms. IEEE Trans. Signal Processing, 42(5):1038-1051, 1994.

[27] D. Q. Mayne, J. B. Rawlings, C. V. Rao \& P. Scokaert. Constrained model predictive control: Stability and optimality. Automatica, 36(6):789-814, 2000

[28] F. Morel, J.-M. Rétif, X. Lin-Shi, B. Allard \& P. Bevilacqua. A predictive control for a matrix converter-fed permanent magnet synchronous machine. In Proc. IEEE Power Electronics Specialists Conf., pp. 1521, Rhodes, Greece, 2008.

[29] T. Perez \& T. I. Fossen. Motion control of marine craft. In W. S. Levine, editor, The Control Handbook: Control System Applications, chapter 32. CRC Press, 2 edition, 2011.

[30] S. J. Qin \& T. A. Badgwell. A survey of industrial model predictive control technology. Control Engineering Practice, 11:733-764, 2003.

[31] D. E. Quevedo \& G. C. Goodwin. Control of EMI from switch-mode power supplies via multi-step optimization. In Proc. AACC American Control Conf., pp. 390-395, Boston, MA, USA, 2004.

[32] J. T. Scruggs \& H. P. Gavin. Earthquake response control for civil structures. In W. S. Levine, editor, The Control Handbook: Control System Applications, chapter 30. CRC Press, 2 edition, 2011.

[33] T. Singh. Optimal Reference Shaping for Dynamical Systems: Theory and Applications. CRC Press, 2009.

[34] G. Strang. The Discrete Cosine Transform. SIAM Review, 41(1):135147, 1999.

[35] G. Strang. Linear Algebra and its Applications. Thomson, 2006.

[36] Y. Tang \& R. Ortega. Adaptive tuning to frequency response specification. Automatica, 29(6):1557-1563, 1993.

[37] D. N. Walker. Torsional Vibration of Turbo-Machinery. McGraw-Hill, 2003. 